TRANSACTIONS OF THE

AMERICAN MATHEMATICAL SOCIETY

Volume 224, Number 2, 1976

\title{
A DECOMPOSITION OF WEIGHTED TRANSLATION OPERATORS
}

\author{
BY
}

JOSEPH J. BASTIAN $\left({ }^{1}\right)$

\begin{abstract}
Let $(X, A, m)$ be a measure space and $T$ an invertible measure-preserving transformation on $X$. Given $\phi$ in $L^{\infty}(X)$, define operators $M_{\phi}$ and $U_{T}$ on $L^{2}(X)$ by $\left(M_{\phi} f(x)=\phi(x) f(x)\right.$ and $\left(U_{T} f\right)=f(T x)$. Operators of the form $M_{\phi} U_{T}$ are called weighted translation operators. In this paper it is shown that every weighted translation operator on a sufficiently regular measure space an be decomposed into a direct integral of weighted translation operators where almost all of the transformations in the integrand are ergodic. It is also shown that every hyponormal weighted translation operator defined by an ergodic transformation is either normal or unitarily equivalent to a bilateral weighted shift. These two results along with some results concerning direct integrals of hyponormal and subnormal operators are used to show that every hyponormal (resp. subnormal) weighted translation operator is unitarily equivalent to a direct integral of normal operators and hyponormal (resp. subnormal) bilateral weighted shifts. The paper concludes with an example.
\end{abstract}

1. Introduction. Weighted shifts, both unilateral and bilateral, have played a fundamental role in Hilbert space operator theory. Primarily, they have been used for examples and counterexamples to illustrate many properties of operators. Shifts can be thought of as infinite but discrete actions on a Hilbert space of square-integrable functions on a measure space. The action can be described in terms of the shift $n \rightarrow n+1$ on the measure space of the integers. If this shift is replaced by a measure-preserving transformation on a more interesting measure space, then the theory of weighted shifts becomes the theory of "weighted translations." It is to be hoped that this collection of operators will be as fruitful a source of research as weighted shifts.

The study of weighted translation operators was initiated fairly recently by Parrott [9]. In his thesis he discussed the spectral properties, numerical ranges, and invariant subspace structure of weighted translation operators. Many of his theorems were based on the hypothesis that the transformation was ergodic.

Presented to the Society, January 15, 1974; received by the editors March 19, 1974. 47A65, 47B99.

AMS (MOS) subject classifications (1970). Primary 47B20, 47A35; Secondary

Key words and phrases. Hyponormal and subnormal operator, weighted shifts, direct integrals of operators, measurable transformation.

$\left(^{1}\right)$ This paper is part of a Ph.D. dissertation written under Professor Paul R. Halmos at Indiana University, 1973. 
The main result of this paper is that every weighted translation operator on a sufficiently regular measure space can be decomposed into a direct integral of weighted translation operators where almost all of the transformations in the integrand are ergodic. This result is achieved by an application of a theorem of Halmos on a direct integral decomposition of a measurable transformation into ergodic transformations. This direct integral decomposition of measure spaces yields a direct integral decomposition of the Hilbert space of square-integrable functions defined on the measure spaces. The main theorem is immediate from these two results. As a consequence of the main theorem and auxiliary results on direct integrals of hyponormal and subnormal operators, it is shown that hyponormal (resp. subnormal) weighted translation operators are direct integrals of hyponormal (resp. subnormal) weighted shifts and normal operators. The paper concludes with an example of a class of subnormal weighted translation operators on the measure space of the real line.

As for terminology, Hilbert space means a complete, complex, separable inner product space; subspace means a closed linear manifold; operator means a bounded linear transformation. The inner product on the Hilbert space will be denoted by $($,$) .$

2. Preliminaries. This paper is concerned with the analysis of a collection of operators defined on the Hilbert space $L^{2}(X)$ of complex-valued measurable square-integrable functions on a measure space $(X, A, m)$. The possible pathology of the measure space $(X, A, m)$ is not significant in the study of the operator structure on $L^{2}(X)$. Therefore, it will be assumed that all measure spaces considered possess certain regularity conditions. To begin with, measure spaces will be assumed to be sigma-finite and separable (the Hilbert space $L^{2}(X)$ is separable). Further regularity conditions will be introduced when needed. A few comments on notation are appropriate.

If $E$ and $F$ are sets in $A$, then $E \Delta F=(E-F) \cup(F-E)$. The notation " $E \subset F$ " will mean $m(E-F)=0$. The sets $E$ and $F$ are said to be equivalent, in symbols " $E=F$ ", if $m(E \Delta F)=0$. Two sigma-subalgebras $A_{1}$ and $A_{2}$ contained in $A$ will be called equivalent if to every set $E$ in either one of them there corresponds a set $F$ in the other so that $E=F$. If $E$ is a measurable set, then $\chi_{E}$ will denote its characteristic function.

A complex-valued function $f$ on $X$ is measurable if $f^{-1}(E)$ is in $A$ for every Borel subset $E$ of the complex plane. A transformation $T$ from $X$ to $X$ is measurable if $T^{-1}(E)$ is in $A$ for each set $E$ in $A$. If $A_{1}$ and $A_{2}$ are equivalent sigma-algebras, then it is easy to prove that every function or transformation which is measurable with respect to $A_{1}$ is equal almost everywhere to one which is measurable with respect to $A_{2}$. 
A transformation $T$ on $X$ is measure-preserving if $m\left(T^{-1} E\right)=m(E)$ for each measurable set $E$. If $T$ is one-to-one and onto, and $T^{-1}$ is measurable, then $T$ is said to be invertible. The symbol $T$ will always refer to a measurable, invertible measure-preserving transformation.

If $f$ is a measurable function on $X$ and $T$ is a transformation, then $f T$ will denote the measurable function whose value at each point $x$ is $f(T x)$. If $T$ is measure-preserving, $f$ is integrable, and $E$ is a measurable set, then

$$
\int_{E} f d m=\int_{T^{-1}} f T d m=\int_{T E} f T^{-1} d m
$$

A transformation $T$ is ergodic if, for any measurable set $E$, the relation $T^{-1} E=E$ implies $m(E)=0$ or $m(X-E)=0$. It is well known that a transformation $T$ is ergodic if and only if the only measurable invariant functions are the constant functions $[6$, p. 25].

An operator $A$ on a Hilbert space $\mathfrak{W}$ is hyponormal if $A^{*} A-A A^{*}$ is positive. An operator is subnormal if it has a normal extension. More precisely, an operator $A$ on a Hilbert space $\mathfrak{W}$ is subnormal if there exists a normal operator $B$ on a Hilbert space $\Re$ such that $\mathfrak{W}$ is a subspace of $\Re$, the subspace $\$$ is invariant under the operator $B$, and the restriction of $B$ to $\mathfrak{W}$ coincides with $A$. It is well known that the subnormal operators form a proper subset of the hyponormal operators [7, Problem 160].

3. Weighted translation operators. Let $(X, A, m)$ be a measure space and $T$ a transformation on $X$. The transformation $T$ defines a unitary operator $U_{T}$ on $L^{2}(X)$ by $U_{T} f=f T$ for $f$ in $L^{2}(X)$. It is easy to see that $\left(U_{T}\right)^{*}=U_{T^{-1}}$, the unitary operator induced by $T^{-1}$. A bounded measurable function $\phi$ in $L^{\infty}(X)$ defines a multiplication operator $M_{\phi}$ on $L^{2}(X)$ by $M_{\phi} f=\phi f$ for $f$ in $L^{2}(X)$. Operators of the form $M_{\phi} U_{T}$ are called weighted translation operators. The weighted translation operator $M_{\phi} U_{T}$ will be denoted by $A_{\phi, T}$, and, when the transformation $T$ is fixed, by $A_{\phi}$. Thus, for $f$ in $L^{2}(X),\left(A_{\phi, T} f\right)(x)=$ $\phi(x) f(T x)$. For a general reference of weighted translation operators, see [9] .

An interesting aspect of weighted translation operators is the "almost" commutativity of $M_{\phi}$ and $U_{T}$, namely $M_{\phi} U_{T}=U_{T} M_{\phi T^{-1}}$. From this it follows that

$$
\left(A_{\phi, T}\right)^{n}=M_{\phi} \cdot \phi T \cdots \phi T^{n-1} U_{T^{n}},
$$

and

$$
\left(A_{\phi, T}\right)^{*}=M_{\phi T^{-1}} U_{T^{-1}}
$$

where $\bar{\phi}$ denotes the complex conjugate of $\phi$. Thus, $A_{\phi, T}$ is normal if and only 
if $\left|\phi T^{-1}\right|=|\phi|$ a.e., and $A_{\phi, T}$ is hyponormal if and only if $\left|\phi T^{-1}\right| \geqslant|\phi|$ a.e.

A transformation $T$ is periodic of period $n$ if $T^{n}=I$ (the identity transformation) and $n$ is the least positive integer for which this occurs. It is easy to see that in the periodic case, the hyponormality of $A_{\phi, T}$ implies normality. However, weaker conditions yield the same result.

THEOREM 3.1. Let $A_{\phi, T}$ be a weighted translation operator on $L^{2}(X)$ where $m(X)<\infty$. If $A_{\phi, T}$ is hyponormal, then it is normal.

Proof. Recall $A_{\phi}\left(=A_{\phi, T}\right)$ is hyponormal if and only if $|\phi| \leqslant\left|\phi T^{-1}\right|$ a.e. Since $m(X)<\infty$, it follows that $|\phi|$ and $\left|\phi T^{-1}\right|$ are integrable positive functions. Since $T$ is measure-preserving, $\int f d m=\int f T^{-1} d m$ for all integrable functions $f$. Hence $\left|\phi T^{-1}\right|-|\phi|$ is a positive integrable function whose integral is zero. Thus $\left|\phi T^{-1}\right|=|\phi|$ a.e. and $A_{\phi}$ is normal.

If $T$ is ergodic, then the only normal weighted translation operators are scalar multiples of unitary operators. The condition $|\phi|=\left|\phi T^{-1}\right|$ a.e. implies, by the ergodicity of $T$, that $|\phi|$ is constant almost everywhere. Multiply by a suitable scalar and assume $|\phi|=1$ a.e. This implies that $M_{\phi}$ is unitary. Since $U_{T}$ is also unitary, it is obvious that $A_{\phi, T}$ is unitary.

4. Direct integrals of Hilbert spaces and operators. In order to present the decomposition of an arbitrary weighted translation operator, some of the theory of direct integrals of Hilbert spaces is required. The presentation of Dixmier [3, Chapter II] appears to lend a clearer correlation between the theory of direct integrals of Hilbert spaces and direct integrals of measure spaces. This correlation will be presented in $\S 5$. The following reference material is presented here. Further exposition on direct integral theory may be found in Dixmier.

Let $(X, A, m)$ be a measure space. Assume that to every $x$ in $X$ there corresponds a separable Hilbert space $\$(x)$. Let $F$ denote the collection of all vector-valued functions defined on $X$ whose value at each $x$ in $X$ is in $\$(x)$.

A sequence $\left\{\gamma_{n}\right\}$ contained in $F$ is said to be a fundamental collection of vector-valued functions if it possesses the following two properties.

(i) The numerical-valued functions $x \rightarrow\left(\gamma_{i}(x), \gamma_{j}(x)\right)$ are measurable functions of $x$ for all $i, j$;

(ii) For all $x$ the vectors $\left\{\gamma_{n}(x)\right\}$ form a total set in $\bar{\Phi}(x)$. (Recall that a set of vectors is called total if the smallest subspace containing it is all of the Hilbert space.)

Suppose $\left\{\gamma_{n}\right\}$ is a fundamental collection of vector-valued functions. Let $G$ be the set of all vector-valued functions $\xi$ in $F$ such that the numerical-valued functions $x \rightarrow\left(\xi(x), \gamma_{n}(x)\right)$ are measurable for all $n$. Then $G$ is a linear submanifold of $F$ and, if $\xi$ is in $G$, then the function $x \rightarrow\|\xi(x)\|$ is measurable.

The vector-valued function $\xi$ in $G$ is said to be square-integrable if 
$\int\|\xi(x)\|^{2} d m(x)$ is finite. The collection of all square-integrable vector-valued functions in $G$ is a complex vector space and will be denoted by $\Re$. For $\xi, \zeta$ in $\Re$ the function $x \rightarrow(\xi(x), \zeta(x))$ is integrable and with respect to the inner product defined by $(\xi, \zeta)=\int(\xi(x), \zeta(x)) d m(x), \Re$ becomes a complex pre-Hilbert space. Thus $\|\xi\|^{2}=\int\|\xi(x)\|^{2} d m(x)$, and $\|\xi\|=0$ if and only if $\|\xi(x)\|=0$ a.e. Upon identification of two elements of $\Re$ if they are equal almost everywhere, the resulting space becomes a Hilbeit space under the obvious operations and inner product, and will be denoted by $\hat{\mathfrak{Q}}=\int^{\oplus} \mathfrak{\Phi}(x) d m(x)$. The Hilbert space $\hat{\bigvee}$ will be called the direct integral of the Hilbert spaces $\$(x)$ over $(X, A, m)$. Actually, $\int^{\oplus} \Phi(x) d m(x)$ depends on the choice of the fundamental set of vectors $\left\{\gamma_{n}\right\}$.

The following statement is important in applications of the direct integral theory. Namely, if $(X, A, m)$ is a separable measure space, then there exists a countable collection $\left\{\xi_{n}\right\}$ contained in $\int^{\oplus} \mathfrak{Q}(x) d m(x)$ such that $\left\{\xi_{n}(x)\right\}$ is a dense subset of $\mathfrak{Q}(x)$ for almost every $\boldsymbol{x}$.

Consider an operator-valued function $A$ on $X$ where, for each $x$ in $X, A(x)$ is an operator on $\$(x)$. The operator-valued function $A$ is said to be measurable if (i) it is defined for almost every $x$ in $X$, (ii) for $\xi$ in $\int^{\oplus} \mathfrak{\$}(x) d m(x)$ the vectorvalued function defined by $x \rightarrow A(x) \xi(x)$ is in $\int^{\oplus} \mathfrak{Q}(x) d m(x)$.

If $A$ is a measurable operator-valued function, then $x \rightarrow\|A(x)\|$ is a measurable numerical-valued function. Furthermore, the measurable operatorvalued function $A$ defines a bounded operator $\hat{A}$ on $\int^{\oplus} \mathscr{F}_{2}(x) d m(x)$ by the formula $(\hat{A} \xi)(x)=A(x) \xi(x)$ if and only if the numerical-valued function $x \rightarrow\|A(x)\|$ is an essentially bounded measurable function. In this case $\|\hat{A}\|$ is the essential supremum of that numerical-valued function.

An opeator $A$ on a direct integral of Hilbert spaces $\int^{\oplus} \Phi(x) d m(x)$ is said to be decomposable if there exists a bounded, measurable operator-valued function $\{A(x)\}$ on $X$ such that $(A \xi)(x)=A(x) \xi(x)$ a.e. for every $\xi$ in $\int^{\oplus} \mathscr{S}(x) d m(x)$. The decomposable operator $A$ will be denoted by $A=\int^{\oplus} A(x) d m(x)$. If $A$ and $B$ are decomposable operators such that $A=\int^{\oplus} A(x) d m(x)$ and $B=\int^{\oplus} B(x) d m(x)$, then for any complex numbers $\alpha, \beta$,

(i) $A^{*}$ is decomposable and $A^{*}=\int^{\oplus} A^{*}(x) d m(x)$;

(ii) $\alpha A+\beta B$ is decomposable and

$$
\alpha A+\beta B=\int^{\oplus}(\alpha A(x)+\beta B(x)) d m(x) ;
$$

(iii) $A B$ is decomposable and $A B=\int^{\oplus} A(x) B(x) d m(x)$.

The remainder of this section presents two results concerning direct integrals of hyponormal and subnormal operators. These results, along with the main decomposition theorem, will be used to classify hyponormal and subnormal weighted translation operators.

It is easy to see that an operator $A$ is hyponormal if and only if $\left\|A^{*} f\right\| \leqslant$ 
$\|A f\|$ for all vectors $f$. Halmos and Bram [2, Theorem 1, p. 76] have shown that an operator $A$ is subnormal if and only if $\Sigma_{i, j}\left(A^{i} f_{j}, A^{j} f_{i}\right) \geqslant 0$ for every finite sequence of vectors $\left\{f_{0}, f_{1}, \ldots, f_{m}\right\}$. It follows easily from these facts that a direct sum, finite or infinite, of operators is hyponormal (subnormal) if and only if each of the summands is hyponormal (subnormal). A similar result will be obtained, with a little more work, for direct integrals of operators.

LEMMA 4.1. Let $\$$ be a separable Hilbert space and $\left\{\xi_{n}\right\}$ a dense subset of $\$$. An operator $A$ on $\$$ is hyponormal if and only if $\left\|A^{*} \xi_{n}\right\| \leqslant\left\|A \xi_{n}\right\|$ for all $n$.

Proof. The necessity is obvious. Sufficiency follows from the continuity of the functions $\xi \rightarrow\left\|A^{*} \xi\right\|$ and $\xi \rightarrow\|A \xi\|$ defined on $\mathfrak{Q}$.

THEOREM 4.2. Let $A=\int^{\oplus} A(x) d m(x)$ be a decomposable operator defined on a direct integral of Hilbert spaces $\hat{\mathfrak{W}}=\int^{\oplus} \mathfrak{S}(x) d m(x)$. The operator $A$ is hyponormal if and only if $A(x)$ is hyponormal for almost every $x$.

Proof. Assume $A$ is hyponormal. There exists a sequence of vectors $\left\{\xi_{n}\right\}$ contained in $\hat{\mathfrak{\$}}$ such that $\left\{\xi_{n}(x)\right\}$ is a dense subset of $\mathfrak{Q}(x)$ for almost every $x$. Discard a set of measure zero and assume that $\left\{\xi_{n}(x)\right\}$ is dense in $\mathfrak{\$}(x)$ for every $x$ in $X$. Let

$$
S_{n}=\left\{x:\left\|A^{*}(x) \xi_{n}(x)\right\|>\left\|A(x) \xi_{n}(x)\right\|\right\},
$$

and $S=\{x: A(x)$ is not hyponormal $\}$. Then $S=\bigcup_{n} S_{n}$. To see this, suppose $x$ is not in $S$. Then $A(x)$ is hyponormal, and by Lemma $4.1 x$ is not in any $S_{n}$. Conversely, if $x$ is in $S$, then $A(x)$ is not hyponormal. Again by Lemma 4.1, there exists a vector $\xi_{n_{0}}(x)$ such that $\left\|A^{*}(x) \xi_{n_{0}}(x)\right\|>\left\|A(x) \xi_{n_{0}}(x)\right\|$. Thus $x$ is in $S_{n_{0}}$.

To complete the proof of necessity, it is enough to show $m\left(S_{n}\right)=0$ for each $n$. Let $\chi_{S_{n}}$ denote the characteristic function of the set $S_{n}$. Let $\zeta_{n}$ denote the vector-valued function defined on $X$ by $\zeta_{n}(x)=\chi_{s_{n}}(x) \xi_{n}(x)$. Since $S_{n}$ is measurable and

$$
\int\left\|\zeta_{n}(x)\right\|^{2} d m(x)=\int_{S_{n}}\left\|\xi_{n}(x)\right\|^{2} d m(x)<\infty,
$$

it follows that $\zeta_{n}$ is in $\hat{\mathfrak{S}}$. Since $A$ is hyponormal,

$$
\begin{aligned}
\left\|A^{*} \zeta_{n}\right\|^{2} & =\int_{S_{n}}\left\|A^{*}(x) \xi_{n}(x)\right\|^{2} d m(x) \\
& \leqslant \int_{S_{n}}\left\|A(x) \xi_{n}(x)\right\|^{2} d m(x) \\
& =\left\|A \zeta_{n}\right\|^{2} .
\end{aligned}
$$


Since $\left\|A^{*}(x) \xi_{n}(x)\right\|^{2}>\left\|A(x) \xi_{n}(x)\right\|^{2}$ for all $x$ in $S_{n}$, it follows that $m\left(S_{n}\right)=0$. Thus $A(x)$ is hyponormal for almost every $x$.

Conversely, if $A(x)$ is hyponormal for almost every $x$, then

$$
\begin{aligned}
\left\|A^{*} \xi\right\|^{2} & =\int\left\|A^{*}(x) \xi(x)\right\|^{2} d m(x) \\
& \leqslant \int\|A(x) \xi(x)\|^{2} d m(x)=\|A \xi\|^{2}
\end{aligned}
$$

for each vector $\xi$ in $\hat{\mathfrak{Q}}$. Hence, $A$ is hyponormal.

The following similar arguments yield the same result for direct integrals of subnormal operators.

LEMMA 4.3. Let $\mathfrak{Q}$ be a separable Hilbert space and $\left\{\xi_{n}\right\}$ a dense subset of $\mathfrak{W}$. An operator $A$ on $\mathfrak{Q}$ is subnormal if and only if $\Sigma_{i, j}\left(A^{i} \xi_{j}, A^{j} \xi_{i}\right) \geqslant 0$ for all finite sequences $\left\{\xi_{0}, \xi_{1}, \ldots, \xi_{m}\right\}$ of vectors contained in the set $\left\{\xi_{n}\right\}$.

Proof. The necessity is obvious. Suppose $\Sigma_{i, j}\left(A^{i} \xi_{j}, A^{i} \xi_{i}\right) \geqslant 0$ for all finite sequences contained in the set $\left\{\xi_{n}\right\}$. Let $\left\{f_{0}, f_{1}, \ldots, f_{m}\right\}$ be a sequence of vectors in $\mathfrak{\$}$. Then each $f_{i}=\lim _{n \rightarrow \infty} \xi_{i, n}$. Thus $A^{j} f_{i}=\lim _{n \rightarrow \infty} A^{j} \xi_{i, n}$ and $\left(A^{i} f_{j}, A^{j} f_{i}\right)=\lim _{n \rightarrow \infty}\left(A^{i} \xi_{j, n}, A^{j} \xi_{i, n}\right)$ for each $i, j=0,1, \ldots, m$. Hence

$$
\sum_{i, j}\left(A^{i} f_{j}, A^{i} f_{i}\right)=\lim _{n \rightarrow \infty}\left(\sum_{i, j}\left(A^{i} \xi_{j, n}, A^{i} \xi_{i, n}\right)\right) \geqslant 0 .
$$

This implies that $A$ is subnormal.

THEOREM 4.4. Let $A=\int^{\oplus} A(x) d m(x)$ be a decomposable operator defined on a direct integral of Hilbert spaces $\hat{\mathfrak{W}}=\int^{\oplus} \mathfrak{Q}(x) d m(x)$. The operator $A$ is subnormal if and only if $A(x)$ is subnormal for almost every $x$.

Proof. Assume $A$ is subnormal. There exists a sequence $\left\{\xi_{n}\right\}$ contained in $\hat{\$}$ such that $\left\{\xi_{n}(x)\right\}$ is a dense subset of $\$(x)$ for almost every $x$. Discard a set of measure zero and assume that the set of vectors $\left\{\xi_{n}(x)\right\}$ is dense in $\mathfrak{P}(x)$ for every $x$ in $X$. Let $\left\{\Xi_{k}\right\}$ be the countable collection of all finite sequences contained in the set $\left\{\xi_{n}\right\}$. Let

$$
S_{k}=\left\{x: \sum_{i, j}\left(A^{i}(x) \xi_{j}(x), A^{j}(x) \xi_{i}(x)\right)<0 ; \xi_{i}, \xi_{j} \in \Xi_{\Xi_{k}}\right\},
$$

and $S=\{x: A(x)$ is not subnormal $\}$.

Then $S=\bigcup_{k} S_{k}$. To see this, suppose $x$ is not in $S$. Then $A(x)$ is subnormal, and by Lemma $4.3, x$ is not in any $S_{k}$. Conversely, if $x$ is in $S$, then $A(x)$ is not subnormal. By Lemma 4.3 there exists a finite sequence $\Xi_{k_{0}}=$ $\left\{\xi_{0}, \xi_{1}, \ldots, \xi_{m}\right\}$ such that $\Sigma_{i, j}\left(A^{i}(x) \xi_{j}(x), A^{j}(x) \xi_{i}(x)\right)<0$. Thus $x$ is in $S_{k_{0}}$.

To complete the proof of necessity, it is enough to show $m\left(S_{k}\right)=0$ for 
each $k$. Assume $\Xi_{k}=\left\{\xi_{0}, \xi_{1}, \ldots, \xi_{m}\right\}$. Let $\zeta_{i}$ be the vector-va'jed function defined by

$$
\zeta_{i}(x)=\chi_{s_{k}}(x) \xi_{i}(x)
$$

for $i=0,1, \ldots, m$. Since $S_{k}$ is a measurable set and $\int\left\|\zeta_{i}(x)\right\|^{2} d m(x)=$ $\int_{S_{k}}\left\|\xi_{i}(x)\right\|^{2} d m(x)<\infty$, it follows that $\zeta_{i}$ is in $\hat{\Phi}$ for each $i$. Since $A$ is subnormal,

$$
\begin{aligned}
\sum_{i, j}\left(A^{i} \zeta_{j}, A^{j} \zeta_{i}\right) & =\sum_{i, j} \int\left(A^{i}(x) \chi_{s_{k}}(x) \xi_{j}(x), A^{j}(x) \chi_{s_{k}}(x) \xi_{i}(x)\right) d m(x) \\
& =\int_{s_{k}}\left(\sum_{i, j} A^{i}(x) \xi_{j}(x), A^{j}(x) \xi_{i}(x)\right) d m(x) \geqslant 0 .
\end{aligned}
$$

However, for $x$ in $S_{k}, \Sigma_{i, j}\left(A^{i}(x) \xi_{j}(x), A^{j}(x) \xi_{i}(x)\right)<0$. This implies $m\left(S_{k}\right)=0$. Hence, $A(x)$ is subnormal for almost every $x$.

Conversely, assume $A(x)$ is subnormal for almost every $x$. Let $\left\{\xi_{0}, \xi_{1}\right.$, $\left.\ldots, \xi_{m}\right\}$ be a finite sequence of vectors in $\hat{\mathfrak{\Phi}}$. Then

$$
\sum_{i, j}\left(A^{i} \xi_{j}, A^{i} \xi_{i}\right)=\int \sum_{i, j}\left(A^{i}(x) \xi_{j}(x), A^{j}(x) \xi_{i}(x)\right) d m(x)
$$

Since $A(x)$ is subnormal for almost every $x$, the function

$$
x \rightarrow \sum_{i, j}\left(A^{i}(x) \xi_{j}(x), A^{j}(x) \xi_{i}(x)\right)
$$

is positive a.e. Since $m$ is a positive measure, it follows that $\Sigma_{i, j}\left(A^{i} \xi_{j}, A^{j} \xi_{i}\right) \geqslant$ 0 , which implies $A$ is subnormal.

5. Main theorem. The primary result of this section, Theorem 5.2, establishes a correlation between the direct integral decomposition of a measure space and a direct integral decomposition of the Hilbert space of square integrable functions over the measure space. The following definition of a direct integral decomposition of a measure space is due to Halmos [4, p. 387].

Definition 5.1. A separable measure space $(\Omega, B, m)$ is said to be decomposable if for every sigma-subalgebra $A$ contained in $B$, the following decomposition exists:

There exists a separable measure space $(X, X, \mu)$ and a disjoint collection $\left\{Y_{x}\right\}_{x \in X}$ of measurable subsets of $\Omega$, indexed by $X$, such that

(i) $\Omega-\bigcup_{x \in X} Y_{x}$ is a set of measure zero $[m]$.

(ii) For each $Y_{x}$ there exists a sigma-finite, separable measure $\nu_{x}$ defined on the induced sigma-algebra $B \cap Y_{x}$ such that for each $B$ in $B$, the function $x \rightarrow \nu_{x}\left(B \cap Y_{x}\right)$ is X-measurable and $m(B)=\int_{X} \nu_{x}\left(B \cap Y_{x}\right) d \mu(x)$. 
(iii) There exists a sigma-algebra $A^{\prime}$ contained in $A$, and equivalent to $A$, with the following property: a subset $B$ of $\Omega$ is in $A^{\prime}$ if and only if $B \cap Y_{x}=$ $Y_{x}$ or $B \cap Y_{x}$ is empty for every $x$ in $X$ and the set $\left\{x: B \cap Y_{x}=Y_{x}\right\}$ is in $X$.

This direct integral decomposition of $\Omega$ will be denoted by $(\Omega, B, m)=$ $\int_{X}^{\oplus}\left(Y_{x}, \nu_{x}\right) d \mu(x)$.

It is known that almost all decent measure spaces are decomposable. Von Neumann [10, Theorem 2, p. 617] has shown that a complete, separable metric space with a regular Borel measure is decomposable. Maharam [8, Theorem 7.2, p. 157], generalizing theorems of Halmos [4, Theorem 1, p. 390] and Ambrose, Halmos and Kakutani [1, Theorem 1, p. 43] has shown that sigma-normal spaces (countable direct sums of measure spaces which are measure-isomorphic to the real line, the unit interval, or the sequence space) are decomposable. The notation used in the following theorem has been introduced above.

THEOREM 5.2. Suppose $(\Omega, B, m)$ is a decomposable measure space. Let $A$ be a sigma-subalgebra contained in $B$ and let

$$
(\Omega, B, m)=\int_{X}^{\oplus}\left(Y_{x}, \nu_{x}\right) d \mu(x)
$$

be the decomposition of $\Omega$ with respect to A. Then the Hilbert space $L^{2}(\Omega)$ can be written as a direct integral of the Hilbert spaces $L^{2}\left(Y_{x}\right)$ over the measure space $(X, X, \mu)$.

Proof. Halmos [4, Lemma 1, p. 387] has shown that if $(\Omega, B, m)$ is a separable measure space, then there exists a sigma-subalgebra $B^{\prime}$ contained in $B$ such that $B^{\prime}$ is countably-generated and $B^{\prime}$ is equivalent to $B$.

Since $(\Omega, A, m)$ is also separable, there exists a sigma-subalgebra $A^{\prime}$ contained in $A$ such that $A^{\prime}$ is countably-generated and $A^{\prime}$ is equivalent to $A$. Let $B^{\prime \prime}$ be the sigma-algebra generated by $B^{\prime}$ and $A^{\prime}$. Then $B^{\prime \prime}$ is countably-generated, $B^{\prime \prime}$ is equivalent to $B$, and $A^{\prime}$ is contained in $B^{\prime \prime}$. Since the Hilbert space structure of $L^{2}(\Omega)$ is not affected by sets of measure zero, it follows that $B$ may be assumed to be countably-generated.

Let $\left\{B_{n}\right\}$ be a sequence of sets that span $B$. Then the sequence of sets $\left\{B_{n} \cap Y_{x}\right\}$ span the induced sigma-algebra in $Y_{x}$. Since $(\Omega, B, m)$ is sigmafinite, it may be assumed that all the $B_{n}$ have finite measure. Since $m\left(B_{n}\right)=$ $\int_{X} \nu_{x}\left(B_{n} \cap Y_{x}\right) d \mu(x)$, it follows that $\nu_{x}\left(B_{n} \cap Y_{x}\right)$ is finite for almost every $x$. Let $A_{n}=\left\{x: \nu_{x}\left(B_{n} \cap Y_{x}\right)=\infty\right\}$. Then $\mu\left(A_{n}\right)=0$. Let $A=\bigcup_{n} A_{n}, X^{\prime}=$ $X-A$. Then $\mu(A)=0$ and $\nu_{x}\left(B_{n} \cap Y_{x}\right)$ is finite for $B_{n}$ in the generating sequence and $x$ in $X^{\prime}$. Since the properties of the decomposition still hold for $X^{\prime}$, it may be assumed that $\nu_{x}\left(B_{n} \cap Y_{x}\right)$ is finite for $B_{n}$ in the generating sequence and every $x$ in $X$. 
It follows from Theorem D, p. 56 of [5] that the smallest subspace of $L^{2}(\Omega)$ which contains the characteristic functions of the generating sets $\left\{B_{n}\right\}$ is all of $L^{2}(\Omega)$. Similar results hold for $L^{2}\left(Y_{x}\right)$ with respect to the characteristic functions of the sets $\left\{B_{n} \cap Y_{x}\right\}$. Let $\int_{X}^{\oplus} L^{2}\left(Y_{x}\right) d \mu(x)$ denote the direct integral of the Hilbert spaces $L^{2}\left(Y_{x}\right)$ where the fundamental set $\left\{\xi_{n}\right\}$ of vector-valued functions is defined by $\xi_{n}(x)=\chi_{\left(B_{n} \cap Y_{x}\right)}$. Since

$$
\left(\xi_{n}(x), \xi_{m}(x)\right)=v_{x}\left(\left(B_{n} \cap B_{m}\right) \cap Y_{x}\right)
$$

for each $x$, it follows from property (ii) of Definition 5.1 that $x \rightarrow\left(\xi_{n}(x)\right.$, $\left.\xi_{m}(x)\right)$ is an $X$-measurable function for every $m$ and $n$, and, from the previous comments, that $\left\{\xi_{n}(x)\right\}$ is a total set in $L^{2}\left(Y_{x}\right)$ for each $x$. Since

$$
\int_{X}\left\|\xi_{n}(x)\right\|^{2} d \mu(x)=m\left(B_{n}\right)<\infty,
$$

it follows that the set $\left\{\xi_{n}\right\}$ is in $\int_{X}^{\oplus} L^{2}\left(Y_{x}\right) d \mu(x)$, and is a total set in $\int_{X}^{\oplus} L^{2}\left(Y_{x}\right) d \mu(x)$.

Let $B$ be in $B$. Then

$$
\begin{aligned}
\int_{\Omega} \chi_{B}(\omega) d m(\omega) & =m(B)=\int_{X} \nu_{x}\left(B \cap Y_{x}\right) d \mu(x) \\
& =\int_{X}\left(\int_{Y_{x}} X_{\left(B \cap Y_{x}\right)}(y) d \nu_{x}(y)\right) d \mu(x) .
\end{aligned}
$$

Thus if $f=\Sigma_{n} \alpha_{n} \chi_{B_{n}}$ is a finite linear combination of characteristic functions of disjoint generating sets $B_{n}$, it is clear that

$$
\begin{aligned}
\int|f(\omega)|^{2} d m(\omega) & =\int\left|\sum_{n} \alpha_{n} \chi_{B_{n}}(\omega)\right|^{2} d m(\omega) \\
& =\int_{X}\left(\int_{Y_{x}}\left|\sum_{n} \alpha_{n} \chi_{\left(B_{n} \cap Y_{x}\right)}(y)\right|^{2} d \nu_{x}(y)\right) d \mu(x) \\
& =\int_{X}\left(\int_{Y_{x}}\left|f_{x}(y)\right|^{2} d \nu_{x}(y)\right) d \mu(x),
\end{aligned}
$$

where $f_{x}$ denotes the restriction of $f$ to $Y_{x}$. For each such $f$ let $U f$ be the vectorvalued function defined by

$$
(U f)(x)=\sum_{n} \alpha_{n} \chi_{\left(B_{n} \cap Y_{x}\right)}
$$

Then $U f$ is an element of $\int_{X}^{\oplus} L^{2}\left(Y_{x}\right) d \mu(x)$ and, from the above remarks, $\|U f\|^{2}=$ $\|f\|^{2}$. It is easy to see that $U$ is a transformation from a dense linear manifold of $L^{2}(\Omega)$ onto a dense linear manifold of $\int_{X}^{\oplus} L^{2}\left(Y_{x}\right) d \mu(x)$, which is obviously linear and norm preserving. Hence, $U$ can be uniquely extended to an isometric isomorphism between $L^{2}(\Omega)$ and $\int_{X}^{\oplus} L^{2}\left(Y_{x}\right) d \mu(x)$. This completes the proof.

The remainder of this section discusses the application of Theorem 5.1 to weighted translation operators. Let $T$ be a transformation on a decomposable 
measure space $(\Omega, B, m)$. A set $E$ in $B$ is said to be invariant if $T E=E$. (Recall the definition of equality of sets in a measure space in $\S 2$.) It is easy to see that the invariant sets of $T$ form a sigma-subalgebra of $B$. The following theorem is a consequence of the previous statement and the fact that $(\Omega, B, m)$ is decomposable.

THEOREM 5.3. If $(\Omega, B, m)$ is a decomposable measure space and $T$ is a measurable, invertible measure-preserving transformation on $\Omega$, then there exists a direct integral decomposition

$$
(\Omega, B, m)=\int_{X}^{\oplus}\left(Y_{x}, v_{x}\right) d \mu(x)
$$

of $(\Omega, B, m)$ in such a way that each $Y_{x}$ is invariant under $T$ and, except possibly for a set of $\mu$ measure zero, $T$ when restricted to $Y_{x}$ is an ergodic, measurable, invertible measure-preserving transformation on $Y_{x}$.

Proof. See [4, Theorem 2, p. 391].

It is this decomposition which has an important application to weighted translation operators. Let $(\Omega, B, m)$ be a decomposable measure space and $A_{\phi, T}$ a weighted translation operator on $L^{2}(\Omega)$. Let $(\Omega, B, m)=$ $\int_{X}^{\oplus}\left(Y_{x}, \nu_{x}\right) d \mu(x)$ be a direct integral decomposition of $\Omega$ into the invariant measure spaces $\left(Y_{x}, \nu_{x}\right)$ on which $T$ is ergodic. Let $T_{x}$ denote the restriction of $T$ to $Y_{x}$. By Theorem 5.2, $L^{2}(\Omega)$ can be represented as the direct integral of the Hilbert spaces $L^{2}\left(Y_{x}\right)$, i.e., $L^{2}(\Omega)=\int_{X}^{\oplus} L^{2}\left(Y_{x}\right) d \mu(x)$. With all the notation established, the following theorem is almost immediate.

THEOREM 5.4. Every weighted translation operator $A_{\phi, T}$ on a decomposable measure space $(\Omega, B, m)$ may be written as a decomposable operator $\int_{X}^{\oplus} A_{\phi_{x}}, T_{x}(x) d \mu(x)$ on a direct integral of Hilbert spaces $\int_{X}^{\oplus} L^{2}\left(Y_{x}\right) d \mu(x)$ such that for almost every $x, A_{\phi_{x}}, T_{x}(x)$ is a weighted translation operator on $L^{2}\left(Y_{x}\right)$, where $T_{x}$ is an ergodic, measurable, invertible measure-preserving transformation on $Y_{x}$.

Proof. Since $\phi$ is actually an equivalence class of functions, it may be assumed that $\phi$ is finite everywhere. For each $x$, the restriction of $\phi$ to $Y_{x}$ is a bounded measurable function $\phi_{x}$ on $\left(Y_{x}, \nu_{x}\right)$. Let $A_{\phi_{x}, T_{x}}$ denote the weighted translation operator defined on $L^{2}\left(Y_{x}\right)$ by $\phi_{x}$ and $T_{x}$. Then the operator-valued function $x \rightarrow A_{\phi_{x}, T_{x}}$ is obviously measurable and since each $\phi_{x}$ is bounded by $\phi$, it follows that this operator-valued function defines a decomposable operator on $\int_{X}^{\oplus} L^{2}\left(Y_{x}\right) d \mu(x)$. It is easy to see that this decomposable operator is equivalent to $A_{\phi, T}$ and the theorem is proved. 
6. Consequences. As a consequence of Theorems 5.4, 4.2 and 4.4, the problem of determining which weighted translation operators are hyponormal (resp. subnormal) has been reduced to analyzing the ones generated by ergodic transformations. However, it will be shown that there are not very many of these.

Let $(X, B, m)$ be a measure space. An atom of the measure space is a measurable set $E$ such that $m(E)>0$ and if $F$ is a measurable set contained in $E$ then $m(F)=0$ or $m(E-F)=0$.

The atoms of a measure space play an important role in the analysis of an ergodic transformation defined on the measure space. The following proposition, which is well known, is an indication of this.

Proposition 6.1. If $(X, B, m)$ is a measure space, $T$ is an ergodic transformation on $X$, and $E$ is an atom, then either

(i) there exists a positive integer $n$ such that $X=\bigcup_{j=0}^{n-1} T^{j} E$ and $m\left(T^{k} E \cap T^{m} E\right)=0$ for $0 \leqslant k<m \leqslant n-1$, or

(ii) $X=\bigcup_{j=-\infty}^{\infty} T^{j} E$ and $m\left(T^{k} E \cap T^{m} E\right)=0$ for $-\infty<k<m<\infty$.

Proof. Suppose that there exists a positive integer $n$ such that $T^{n} E=E$; let $n$ be the smallest positive integer satisfying this condition. Then it follows that $X$ is the union of the pairwise disjoint sets $\left\{T^{j} E\right\}_{j=0}^{n-1}$. The sets are pairwise disjoint (their intersection has zero measure) because $E$ is an atom and $n$ is the least positive integer satisfying $T^{n} E=E$. Since $\bigcup_{j=0}^{n-1} T^{j} E$ is an invariant set of positive measure, it follows that $X=\bigcup_{j=0}^{n-1} T^{j} E$.

If no such positive integer $n$ exists, then for essentially the same reasons $X$ is, except possibly for a set of measure zero, the union of the pairwise disjoint sets $\left\{T^{j} E\right\}_{-\infty}^{\infty}$.

It is easy to see that if $E$ is an atom and $T$ is measure-preserving, then $T^{j} E$ is an atom for all integers $j$. Suppose $A_{\phi, T}$ is a weighted translation operator defined on $L^{2}(X)$. Since any measurable function is constant on an atom, it follows that in the first case of the proposition, $A_{\phi, T}$ is a finite-dimensional weighted translation operator, and in the second case, $A_{\phi, T}$ is a bilateral weighted shift. Since the transformation $T$ in the finite-dimensional case is periodic, any such hyponormal weighted translation operator must be normal. Actually, it is known that any hyponormal operator on a finite-dimensional space is normal [7, Problem 159]. Hence, the problem has been reduced to studying hyponormal (subnormal) weighted translation operators on nonatomic measure spaces. It will be shown that these must be normal. This fact is derived from the following lemma which is well known to ergodic theorists. See [6, p. 85] for a proof.

LEMMA 6.2. If $(X, B, m)$ is a measure space with only finitely many 
atoms, $T$ is an ergodic transformation on $X$, and if $E$ is a measurable set such that $T E \subset E$, then $m(E)=0$ or $m(X-E)=0$.

THEOREM 6.3. If $T$ is an ergodic transforamtion on a nonatomic measure space, then every hyponormal weighted translation operator $A_{\phi}$ determined by $T$ is normal.

Proof. Since $A_{\phi}$ is hyponormal, $\left|\phi T^{-1}\right| \geqslant|\phi|$ a.e. Let $M$ denote the essential supremum of $\phi$. It may be assumed that $M>0$. Let $\alpha$ be any positive number strictly less than $M$. Let $E=\{x:|\phi(x)| \geqslant \alpha\}$. Then $m(E)$ is not zero and $\left|\phi T^{-1}\right| \geqslant|\phi|$ a.e. implies $T^{-1} E \subset E$. By Lemma 6.7, $X-E$ is a set of zero measure. Since this is true for arbitrary $\alpha, 0 \leqslant \alpha<M$, it follows that $|\phi|=M$ a.e. Hence, $|\phi|=\left|\phi T^{-1}\right|$ a.e. and $A_{\phi}$ is normal.

The following theorem summarizes the previous results and comments.

THEOREM 6.4. Every hyponormal (subnormal) weighted translation operator on a decomposable measure space is a direct integral of normal operators and hyponormal (subnormal) bilateral weighted shifts.

Proof. This follows directly from Theorems 4.2, 4.4, 5.4, 6.3 and Proposition 6.1. Recall that from the comment after Theorem 3.1, the normal operators in the direct integral are actually scalar multiples of unitary operators.

7. An example. This section analyzes weighted translation operators on the measure space $(R, B, m)$ of the real line with the sigma-algebra $B$ of Borel subsets and Lebesgue measure $m$. The transformation $T$ considered will be defined by $T \omega=\omega-1$. Each bounded measurable function $\phi$ on $R$ defines a weighted translation operator on $L^{2}(R)$ by $\left(A_{\phi} f\right)(\omega)=\phi(\omega) f(\omega-1)$. The decomposition implied by Theorem 5.4 will show that such a weighted translation operator is a direct integral of bilateral weighted shifts. The weight sequences for these weighted shifts are determined by the values of the multiplying function $\phi$ at the points $x, x \pm 1, x \pm 2, \ldots$ for $x$ in the interval $[0,1)$. From this decomposition into a direct integral of weighted shifts, the determination of which such weighted translation operators are hyponormal (resp. subnormal) follows easily from the results of $\S 3$.

Let $A$ denote the sigma-subalgebra of $B$ composed of the invariant sets for $T$. Let $(I, S, \mu)$ denote the Borel measure space of the interval $[0,1)$ with Lebesgue measure $\mu$. For each $x$ in $I$, let $Y_{x}$ be the subset $\left\{T^{n} x\right\}_{-\infty}^{\infty}$ of $R$. For each $x$ in $I$ form the measure space $\left(Y_{x}, B_{x}, \nu_{x}\right)$ where $B_{x}$ is the sigma-algebra of all subsets of $Y_{x}$ and $\nu_{x}$ is the counting measure on $B_{x}$. It is easily verified that the measure spaces $(I, S, \mu)$ and $\left(Y_{x}, B_{x}, \nu_{x}\right)_{x \in I}$ satisfy the conditions of Definition 5.1. Note that if $E$ is in $A$, then $E=\bigcup_{-\infty}^{\infty} T^{n}(E \cap I)$. It follows 
from Theorem 5.4 that the weighted translation operator $A_{\phi, T}$ is unitarily equivalent to a decomposable operator $\int_{I}^{\oplus} A_{\phi_{x}}, T_{x}(x) d \mu(x)$ on the direct integral of the Hilbert spaces $\int_{I}^{\oplus} L^{2}\left(Y_{x}\right) d \mu(x)$. However, upon inspection, it can be seen that the Hilbert spaces $L^{2}\left(Y_{x}\right)$ are isometrically isomorphic to the doubly-infinite sequence space $l^{2}$, and that the weighted translation operators $A_{\phi_{x}}, T_{x}$ on $L^{2}\left(Y_{x}\right)$ are unitarily equivalent to the bilateral weighted shifts on $l^{2}$ with weights $\left\{\phi\left(T^{-n-1} x\right)\right\}_{-\infty}^{\infty}$. Hence, the following theorem is immediate from Theorems 4.2, 4.4, and 5.4.

THEOREM 7.1. A weighted translation operator $A_{\phi, T}$ on $L^{2}(R)$, where $T$ is the measure-preserving transformation $T \omega=\omega-1$, is hyponormal (resp. subnormal) if and only if the bilateral weighted shifts with weights $\left\{\phi\left(T^{-n-1} x\right)\right\}_{-\infty}^{\infty}$ are hyponormal (resp. subnormal) for almost every $x$ in $[0,1)$.

Note that if $\phi$ is a bounded measurable function on the real line such that $|\phi|$ is a nondecreasing function then the weighted translation operator generated by $\phi$ and $T$ is trivially hyponormal.

\section{BIBLIOGRAPHY}

1. W. Ambrose, P. R. Halmos and S. Kakutani, The decomposition of measures. II, Duke Math. J. 9 (1942), 43-47. MR 3, 210.

2. J. Bram, Subnormal operators, Duke Math. J. 22 (1955), 75-94. MR 16, 835.

3. J. Dixmier, Les algèbres d'opérateurs dans l'espace hilbertien, Gauthier-Villars, Paris, 1969. (For a review of the 1st ed., 1957, see MR 20 \#1234.)

4. P. R. Halmos, The decomposition of measures, Duke Math. J. 8 (1941), 386-392. MR 3, 50.

5. - Measure theory, Van Nostrand, Princeton, N. J., 1950. MR 11, 504.

6. Lectures on ergodic theory, Publ. Math. Soc. Japan, no. 3, Math. Soc. of Japan, Tokyo, 1956; reprint, Chelsea, New York, 1960. MR 20 \#3958; 22 \#2677.

7. - A Hilbert space problem book, Van Nostrand, Princeton, N.J., 1967. MR 34 \#8178.

8. D. Maharam, Decompositions of measure algebras and spaces, Trans. Amer. Math. Soc. 69 (1950), 142-160. MR 12, 167.

9. S. K. Parrott, Weighted translation operators, Dissertation, Univ. of Michigan, Ann Arbor, Mich., 1965.

10. J. von Neumann, Zur Operatorenmethode in der Klassischen Mechanik, Ann. of Math. (2) 33 (1932), 587-642.

DEPARTMENT OF MATHEMATICS, WRIGHT STATE UNIVERSITY, DAYTON, 